\title{
Subjective well-being of Sichuan adolescents from Han, Qiang and Yi nationalities*
}

\author{
Dingchu $\mathrm{Wu}^{1 *}$, Tianmei Zhou ${ }^{2}$ \\ ${ }^{1}$ Institute of the Educational Science, Sichuan Normal University, Chengdu, China; *Corresponding Author: wudingchu@sina.com \\ ${ }^{2}$ Institute of Educational Science, Neijiang Normal University, Neijiang, China
}

Received 20 June 2010; revised 5 July 2010; accepted 19 July 2010.

\begin{abstract}
Using subjective well-being scale for adolescents, this article studies the subjective well-being of 900 adolescents from Han, Qiang and Yi nationalities in mainland of China. Results: In general, the SWB of the adolescents from the three nationalities is at the level of more than medium. Their SWB is significant difference in the aspects of nationality, grade and academic records. In view of interaction, their SWB is also of differrent dimensions because the interrelated effects are significant between genders and grades, between genders and academic records, between grades and academic records, and even between grades and their parental educational levels and parental occupations.
\end{abstract}

Keywords: Adolescent; Subjective Well-Being; Nationality; Scale

\section{INTRODUCTION}

Subjective Well-being (SWB) is an important and comprehensive psychological index that is used to measure the quality of life for people [1]. It includes two fundamental components: cognitive component and emotional component. Cognitive component mainly refers to life satisfaction of individuality, including general life satisfaction and specific aspect of life satisfaction. Whereas, emotional component usually refers to emotional experience that one feels in real life, including positive emotions and negative emotions [2]. Since 1976, Wanner Wilson publishing Correlates of Avowed Happiness, the research on SWB in western countries has developed from description stage to construction stage of theory,

\footnotetext{
"Foundation Item: Sichuan Provincial Education Department of humanities and social sciences key research base a funded project in Sichuan "Applied Psychology Research Center, Chengdu Medical College" base (No: CSXL-092003).
}

and now, it is approaching to the deepening stage of theory [3]. However, compared with the research on adult SWB, the research on adolescent SWB is relatively much fewer. Moreover, at present, related work that has been done still mainly focuses on the field of life satisfaction [4]. In China, the research on SWB started in the mid 1980's. Now, the research is in the starting and exploring stage and further empirical study is needed. Note that adolescent SWB is relatively fewer and it mainly develops in two ways: 1) Exploring the well-being scale for adolescents. According to the published documents and applied examples, Life Satisfaction Scale for adolescent, which is mainly formed by Zhang Xing-gui [5] on the base of Huebner's Multidimensional Life Satisfaction Scale for Children (MSLSS), is widely used. On the basis of Life Satisfaction Scale for Young Students, life satisfaction for adolescent includes self satisfaction and environment satisfaction. Self satisfaction consists of four factors, namely friendship satisfaction, family satisfaction, school work satisfaction, and freedom satisfaction; whereas environment satisfaction consists of two factors, namely school satisfaction and social \& natural environment satisfaction. 2) Research on the present situation of SWB for adolescents and influencing factors. It mainly includes social relations, personality traits, and concepts about self. And there is almost no research on different nations' or cross-cultural SWB.

Many intercultural researches have proved that culture has great effect on SWB [6]. China is a multi-ethnic country and almost each nation has its distinctive religious beliefs, traditional customs, and languages. Therefore, it is inevitable to form a lot of different cultural backgrounds in China. But what is the difference of adolescent SWB in different nations? So, discussing the conditions of adolescent SWB in different nations of China can not only enrich and deepen the research of SWB, but also help adolescent to master the strategies to improve SWB and to experience more well-being in specific conditions. In this paper, we compare adolescent SWB of Han nationality, Qiang nationality, and Yi nationality in western 
China and discuss how national elements effected the development and characteristic of adolescent SWB.

\section{METHOD}

\subsection{Participants}

We randomly chose eighteen classes in six grades from junior middle schools to senior middle schools, which included Liangshan where the Yi live together, Beichuan, Mianyang where the Qiang live together, and Ziyang \& Neijiang where the Hans live together. We totally issued 900 questionnaires and regained 895 questionnaires. Removing invalid questionnaires, we got 867 valid questionnaires, including 442 questionnaires of Han students, 231 questionnaires of Qiang students, 193 questionnaires of Yi students; 352 questionnaires of male students, 515 questionnaires of female students; 300 questionnaires of urban students, 565 questionnaires of rural students. The numbers of questionnaires of six grades from Grade 7 to Grade 12 are 122, 148, 134, 139, 232, and 91. Ages of students are from 11 to $19(15.6 \pm 1.6)$.

\subsection{Research Materials}

Subjective Well-being Scale adopts Zhang Xinggui's CMSLSS (China Adolescent Multidimensional Life Satisfaction Scale) [5] which consult Huebner (1994)' MSLSS(Multidimensional Life Satisfaction scale) and Bradburn's Affect Scales [7], CMSLSS which includes six dimensions and thirty seven items, such as school, school work, friendship, family, environment, freedom satisfaction and so on, is chosen to measure adolescent SWB. The homogeneity reliability of CMSLSS varies from 0.87 to 0.93 and the stability reliabilities of total scale and subscales vary from 0.80 to 0.85 during one month. According to published documents and exploratory factor analysis, cumulative variance contribution rate of six factors is $49.54 \%$ and confirmatory factor analysis also proves the construct validity. CMSLSS adopts seven-point system and for each test item, the lowest score is 1 and the highest score is 7 . More score means higher level of life satisfaction. And in our test, for Cronbach's alpha of the CMSLSS, total score is 0.85 . In Affect Scales, there are ten items for true or false, including five items to describe positive emotions and five items to describe negative emotions. Answering "Yes" stands for 1 score and "No" stands for 0 score.

Demographic Variable Questionnaire

This questionnaire is mainly used to collect personal information, including nationality, sex, grade, academic record (outstanding, satisfactory, average, and poor), educational background of parents (college and above, senior middle school, junior middle school and below), profession of parents (labor, peasant, and intellect).

\subsection{Procedure}

Using stratified cluster sampling method and anonymous test by class, we regained questionnaires at once after test. Before test, the examiners directed students how to rightly fill the forms under the instructions. After students had understood rules fully, the test began. The head teacher of each class acted as examiner and was trained in advance.

\subsection{Data Analysis}

In our study, data was processed and analyzed by SPSS for Windows 13.0.

\section{RESULTS}

\subsection{Descriptive Statistics}

The average score of the total of CMSISS for adolescents of Han, Qiang, and Yi nationalities is $4.55 \pm 0.85$. From high to low, the scores of the six dimensions are: family satisfaction $(5.23 \pm 0.86)$, friendship satisfaction (4.96 \pm 0.86$)$, freedom satisfaction (4.64 \pm 0.86$)$, environment satisfaction $(4.45 \pm 0.86)$, school satisfaction $(4.26 \pm 0.86)$, and academic satisfaction $(3.26 \pm 0.87)$. The score of negative emotions $(3.12 \pm 0.84)$ is higher than that of positive emotions $(2.82 \pm 0.84)$. Hence, the score on family satisfaction for adolescents of Han, Qiang, and Yi nationalities is the highest, exceeding average level(4); whereas the score of school work satisfaction is the lowest, below average level(4), with major negative emotions.

\subsection{Comparison of SWB for Adolescents of Han, Qiang, and Yi Nationalities}

Through analysis of variance for adolescents SWB of different nationalities, the result is shown in Table 1. Besides friendship satisfaction, environment satisfaction and positive emotions, the scores on other dimensions among Han, Qiang, and Yi nationalities are obviously different. Based on Table 1, multiple comparisons were proceeding via least significant difference (LSD). The scores on the total of CMSISS and the dimensions of family satisfaction and school satisfaction for adolescents of Yi nationality are obviously higher than that for adolescents of Han and Qiang nationalities $(P<0.01)$. For the dimension of school work satisfaction, the scores for adolescents of Qiang and Yi nationalities are higher than that for adolescents of Han nationality $(P<0.01)$. For the factor of freedom satisfaction, the score for adolescents of Qiang nationality is lower than that for adolescents of Yi nationality $(P<0.05)$. For negative emotions, the scores for adolescents of Yi nationality are more than 
those for adolescents of Qiang and Han nationalities.

\subsection{Relationship between Demographic Variables and SWB for Adolescents of Han, Qiang, and Yi Nationalities}

\subsubsection{Study Results of SWB for Adolescents of Han, Qiang, and Yi Nationalities Related to Sex, Grade, and Academic Record Variables}

Using the factors in CMSLSS and Affect Scales as dependent variables, with MANOVA of such variables sex, grade and academic record (Table 2), the results of Table 2 show that: For main effects, there are obvious differences in friendship satisfaction, school satisfaction, school work satisfaction, and freedom satisfaction between different grade; for academic record (excellent, good, fair and poor), there are also obvious differences in friendship satisfaction, family satisfaction, school satisfaction, school work satisfaction, freedom satisfaction, and negative emotions; for the factors of SWB, the difference in sex main effect is obscure. Considering interactions, for school work satisfaction and environment satisfaction, there are obvious interactions with sex and grade; for sex and academic record, there are also obvious interactions with friendship satisfaction, family satisfaction, school satisfaction, school work satisfaction and freedom satisfaction; for grade and academic record, there are obvious interactions with friendship satisfaction and school work satisfaction; for sex, grade and academic record, there are no interactions with the dimensions of SWB.

Table 1. The comparison of SWB scores for adolescents of Han, Qiang, and Yi nationalities.

\begin{tabular}{|c|c|c|c|c|c|c|c|}
\hline \multirow{2}{*}{ scale } & \multirow{2}{*}{$\begin{array}{c}\text { Han } \\
(\mathrm{N}=442)\end{array}$} & \multirow{2}{*}{$\begin{array}{c}\text { Qiang } \\
(\mathrm{N}=231)\end{array}$} & \multirow{2}{*}{$\begin{array}{c}\mathrm{Yi} \\
(\mathrm{N}=193)\end{array}$} & \multirow{2}{*}{$F$} & \multicolumn{3}{|c|}{ post-hoc comparisons $(P<0.01)$} \\
\hline & & & & & (1)(2) & (1)(3) & (2)(3) \\
\hline family satisfaction & $5.15 \pm 1.26$ & $5.17 \pm 1.19$ & $5.56 \pm 1.10$ & $8.23^{* * *}$ & & (1) < (3) & (2) $<$ (3) \\
\hline friendship satisfaction & $5.01 \pm 0.82$ & $4.89 \pm 0.81$ & $4.95 \pm 0.73$ & 1.91 & & & \\
\hline school satisfaction & $4.20 \pm 0.73$ & $4.23 \pm 0.74$ & $4.43 \pm 0.74$ & $6.84^{* *}$ & & (1) $<$ (3) & (2) $<$ (3) \\
\hline school work satisfaction & $3.48 \pm 1.16$ & $3.73 \pm 1.10$ & $3.80 \pm 1.06$ & $6.95^{* *}$ & (1) $<$ (2) & (1) $<$ (3) & \\
\hline freedom satisfaction & $4.70 \pm 1.19$ & $4.47 \pm 1.08$ & $4.69 \pm 1.03$ & $3.49^{*}$ & & & (2) $<$ (3) \\
\hline environment satisfaction & $4.46 \pm 0.88$ & $4.40 \pm 0.81$ & $4.47 \pm 0.88$ & 0.49 & & & \\
\hline Negative emotion & $3.02 \pm 1.43$ & $3.04 \pm 1.44$ & $3.49 \pm 1.33$ & $7.92^{* * *}$ & & (1) < (3) & (2) $<$ (3) \\
\hline
\end{tabular}

Note: ${ }^{*} P<0.05 ;{ }^{* *} P<0.01 ;{ }^{* * *} P<0.001$.

Table 2. ANOVA of sex, grade and academic record in SWB $(F)$.

\begin{tabular}{|c|c|c|c|c|c|c|c|}
\hline Variation Source & $\mathrm{a}$ & $b$ & $\mathrm{c}$ & $a \times b$ & $\mathrm{a} \times \mathrm{c}$ & $\mathrm{b} \times \mathrm{c}$ & $\mathrm{a} \times \mathrm{b} \times \mathrm{c}$ \\
\hline the total of CMSISS & 0.04 & 1.51 & $3.28^{*}$ & 1.12 & 2.01 & 0.86 & 0.93 \\
\hline friendship satisfaction & 2.92 & $2.38^{*}$ & $13.58^{* * *}$ & 1.17 & $3.36^{*}$ & $2.10^{* *}$ & 1.38 \\
\hline family satisfaction & 0.57 & 0.50 & $7.11^{* * *}$ & 2.19 & $3.36^{*}$ & 0.93 & 0.65 \\
\hline school satisfaction & 0.38 & $3.28^{* *}$ & $2.93^{*}$ & 1.59 & $3.53^{*}$ & 1.00 & 1.23 \\
\hline school work satisfaction & 0.01 & $11.03^{* * *}$ & $13.65^{* * *}$ & $2.41^{*}$ & $3.54^{*}$ & $2.32^{* *}$ & 1.44 \\
\hline freedom satisfaction & 0.12 & $3.45^{*}$ & $3.26^{*}$ & 1.82 & $3.09^{*}$ & 1.60 & 1.23 \\
\hline Environment satisfaction & 0.94 & 1.14 & 1.98 & $2.53^{*}$ & 0.62 & 1.16 & 1.18 \\
\hline positive emotion & 1.88 & 2.22 & 1.01 & 0.84 & 0.80 & 1.49 & 1.00 \\
\hline Negative emotion & 0.00 & 1.36 & $3.70^{*}$ & 0.98 & 0.35 & 1.62 & 0.88 \\
\hline
\end{tabular}

Note: a: gender, b: grade, c: academic record.; $* P<0.05 ; * * P<0.01 ; * * * P<0.001$. 
According to Figure 1, with the increase of grade, the trend of the scores on the total of CMSISS and the dimensions of school satisfaction, school work satisfaction, and negative emotions declines. The scores on friendship satisfaction and freedom satisfaction vary like wave crest and general trend is upward, from Grade7 to Grade 10 , in which score of Grade 11 and Grade 12 is slightly lower than that of Grade 10, and the scores of senior middle school are higher than those of junior middle school. With the increase of grade, trend of the scores on positive emotions is upward, too; trends of the scores on family satisfaction and environment satisfaction are nearly linear and parallel. According to Figure 2, for academic record, except environment satisfaction, better of academic record leads to higher of the scores on other dimensions of SWB. In multiple comparison, through LSD, we find that except environment satisfaction, life satisfaction of adolescents with excellent or good academic record is obviously higher than that of adolescents with average or poor academic record $(P<0.05)$, and Negative emotions of adolescents with excellent or good academic record are much higher than those with average or poor academic record $(P<0.01)$.

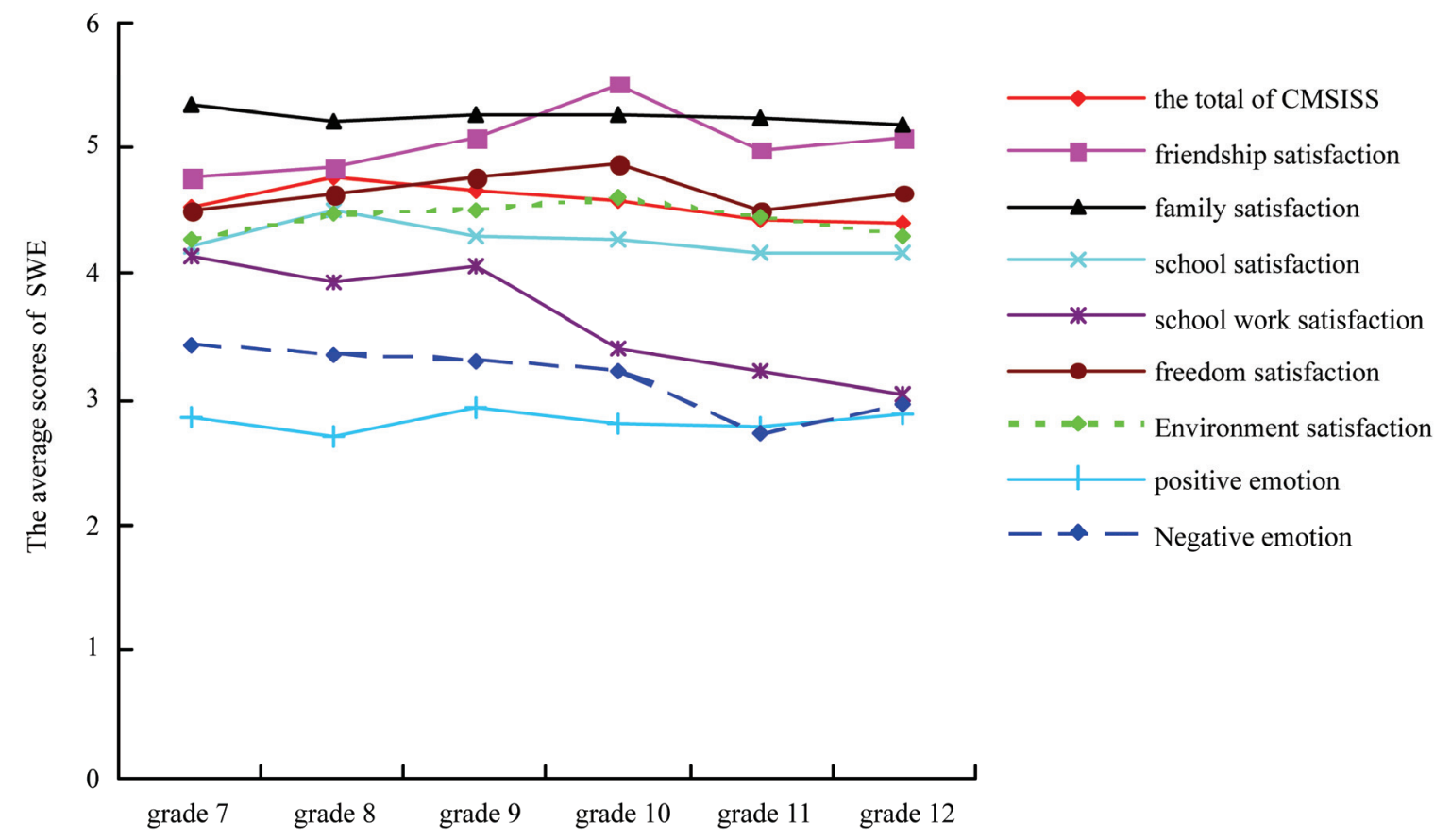

Figure 1. The trends of SWB scores for adolescents in different grade.
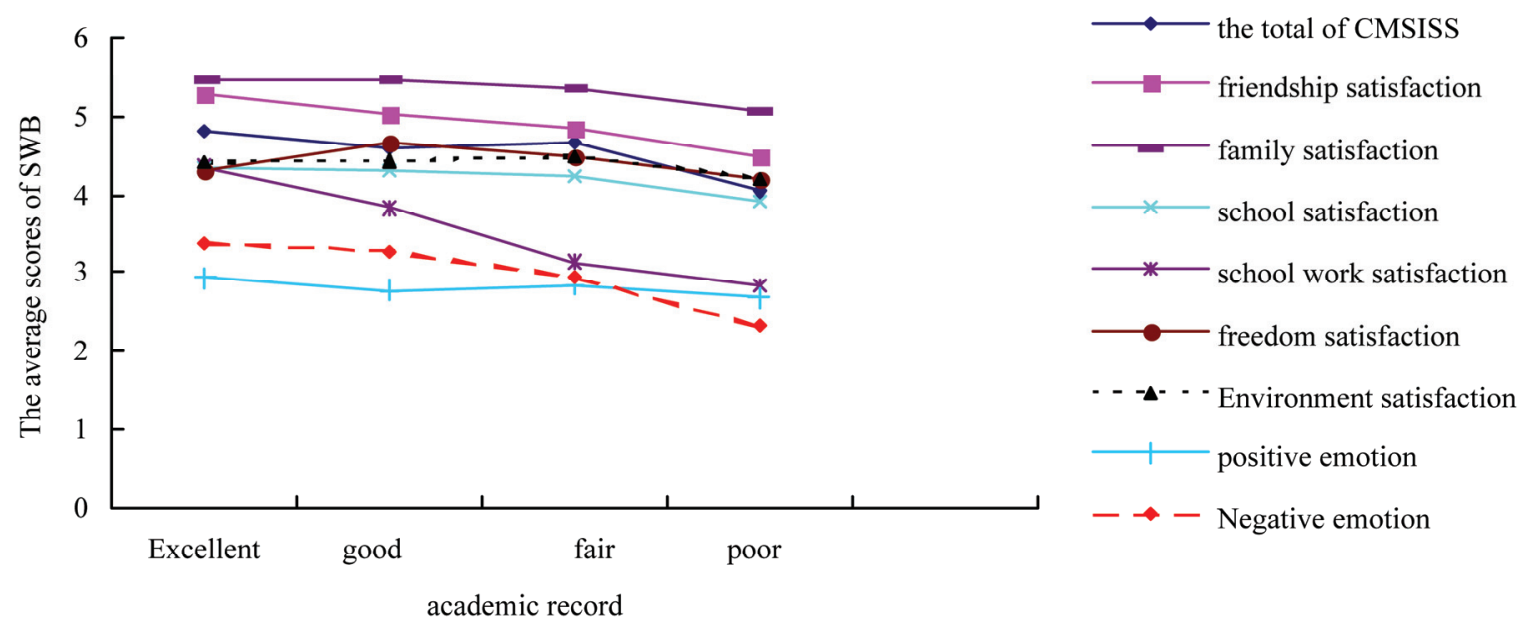

Figure 2. The trends of SWB scores for adolescents in different academic record. 


\subsubsection{Study Results of SWB for Adolescents of Han, Qiang, and Yi Nationalities Related to Education Background and Profession Variables of Parents}

Using the factors in CMSLSS and Affect Scales as dependent variables, with MANOVA of such variables educational background and profession of parents .The results show that only environment satisfaction has main effect with Mother's profession $(F=4.40, P<0.05)$ and the other dimensions of SWB have no main effects with education background and profession of parents. School satisfaction has obvious interactions with education background and profession of mother $(F=2.53, P<0.05)$, profession of father $(F=2.65, P<0.05)$, education background and profession of father $(F=3.66, P<0.05)$, and education background of father and profession of parents $(F=3.25, P<0.05)$. Friendship satisfaction has obvious interactions with education background of mother and profession of parents $(F=4.52, P<0.01)$. Family satisfaction has obvious interactions with profession of parents $(F=3.54, P<0.01)$, education background of mother, profession of parents $(F=6.01, P<0.001)$. Negative emotions has obvious interactions with profession of parents $(F=2.65, P<0.05)$.

Through multiple comparison based on LSD, we can draw conclusions that for adolescents whose mothers are with senior middle school education background, school satisfaction is obviously lower than those whose mothers are with junior middle school or lower education background $(P<0.05)$; for adolescents whose parents are with senior middle school education background or are intellects, friendship satisfaction is obviously higher than those whose parents are with junior middle school education background or are Farmers $(P<0.05)$; for adolescents whose parents are Farmers, family satisfaction is obviously higher than those whose fathers are labors or mothers are intellects $(P<0.05)$; for adolescents whose mothers are with college education background, positive emotions are obviously higher than those whose mothers are with junior middle school education background $(P$ $<0.05$ ); for adolescents whose fathers are intellects, negative emotions are obviously lower than those whose fathers are Farmers $(P<0.05)$.

\section{DISCUSSIONS}

\subsection{Fundamental State of SWB for Adolescents of Han, Qiang, and Yi Nationalities}

According to our research, the general life conditions for adolescents of Han, Qiang, and Yi nationalities are rath- er satisfying and in the dimensions of SWB, for adolescents, family satisfaction and friendship satisfaction are highest and school work satisfaction is lowest, with rather more negative emotions. So the level of SWB for adolescents is higher than the average level and it is roughly consistent with the domestic and international research result [8-10]. We can draw conclusions that Chinese adolescents can get a lot of supports and acceptances from their parents and with the increase of ages, adolescents can comprehend and understand friendship deeply and fully. So the friendship becomes more stable and deeper. Nevertheless, the lowest school work satisfaction and rather more negative emotions may have close relationship with study pressure of adolescents.

\subsection{Difference of SWB Related to Nationality}

According to our study, nationality acts as an important factor in SWB of adolescents. For adolescents of Yi nationality, the total of CMSISS and negative emotions are obviously higher than those of adolescents for Han, Qiang nationalities. For adolescents of Qiang and Yi nationalities, school work satisfaction is higher than that of adolescents for Han nationality. For adolescents of Yi nationality, freedom satisfaction is obviously lower than that of adolescents for Qiang nationality. Maybe different nationality populations stand for different sets of cultures, social norms, values, socioeconomic statuses, family parenting patterns, partner attitude and school educations. And these different sets constitute of the social background or social reference group, namely mainstream attitude, for adolescents to estimate SWB, reflecting the intercultural differences of SWB. A lot of related empirical study has proved that culture is an important factor of SWB. Many researchers believe that different individuals in different cultures have differences in life satisfaction, emotion satisfaction, social orientation, value, and judgment criterion [6]. For example, the Hans with edification of Confucianism believe in that the worth of other pursuits is small, and the study of books excels them all. So many people treat the study of books as the only way to success. Young students bear too many things for entering college and in elementary schools, junior middle schools, or senior middle schools, parents, teachers and friends all ground the idea into their heads that only by entering colleges they will be promising. Therefore, study and school work become the goals of adolescents for Han nationality. If they fail or do not do well as expected, adolescents have to bear criticism and blame from many sides and it surely will lead to lower school work satisfaction compared with adolescents of Qiang and $\mathrm{Yi}$ nationalities. 


\subsection{Difference Analysis of SWB of Adolescents for Han and Qiang Nationalities Based on Demographic Variables}

In our study, there are great differences of SWB of adolescents for different grade and it is not consistent with the result of foreign research [11-13]. But it is consistent with the result of domestic research [14-17]. At the same time, for academic record, it has great effect on SWB of adolescents and this conclusion supports the existing domestic research $[15,16,18]$. For Chinese adolescents, with the increasing of grade, school satisfaction and school work satisfaction decrease, and friendship satisfaction and freedom satisfaction rise and fall in turn. Besides environment satisfaction, life satisfaction of adolescents with better academic records is obviously higher than that of adolescents with average or poor academic records. And the same condition appears again for negative emotions. It has close relation with study pressure that Chinese adolescents face with. According to related researches, the greatest source of pressure for middle school students is school work [19]. Adolescence is also at the stage of middle school days in which Chinese adolescents are most concerned with the study exercises of themselves and their important goal and mission are to get better academic records. So that academic record affects the self-evaluations and general assessments of life quality for adolescents. When adolescents in Grade 7 enter a new environment, they will experience many new things, namely new start of school work, new teachers and classmates, and all are exciting and expecting. There will be a long time for Grade 7 or 8 to face entrance examinations for senior middle schools or universities and study pressures are relatively lowest. Therefore, in this stage, the dimensions of SWB for adolescents are relatively higher. With the increase of grade, the difficulty and pressure of study obviously increase, too. It will cause adolescents to treat school work as a kind of burdens, not a necessary need for them. Moreover, it will finally cause polarization of academic records. On the other hand, with the approach of entrance examination or employment pressure, adolescents have to bear huge psychological pressure. First of all, under the pressure from school, family, and society, adolescents have to take entrance examinations for senior middle schools or universities seriously and the pleasure and freedom of study disappear because of educational utilitarianism. Then, the exorbitant expectation from parents will force the senior students to put more time and energy into their study. So students with average or poor academic records will face with stricter control and restriction, at the same time, it will lead school satisfaction, school work satisfaction of adolescents to decrease, and friendship satisfaction and freedom satisfaction of adolescents to vary up and down in turn. Because adolescents with better academic records may get more supports from family and acceptances from teachers and classmates, life satisfaction of adolescents with better academic records is higher than that of adolescents with poor academic records. However, too high goals or conflicts among different goals will generate more negative emotions for adolescents with better academic records than the adolescents with poor academic records have less or no goals. It is surprising that in the SWB of adolescents, positive emotions increase slowly. The reason may be that positive emotions are the buffering factors in SWB of adolescents. When SWB and its factors decrease rapidly, the increase of positive emotions is helpful for the development of mental health and psychological quality, and for adolescents to adapt to study and life. So, academic pressure is the main source of pressures that Chinese adolescents are facing and it affects not only mental health but also experience of happiness of adolescents, with negative effect on life quality of adolescents.

In this paper, our results suggest that the factors of education background and profession of parents have important effects on SWB of adolescents. This effect may have relationship with parenting styles caused by different education backgrounds and professions of parents. The research made by Hu Jie, Ji Tian-shu, Feng Fenglian shows college students' SWB has positive relation to parents' warmth and understanding in parenting and has obvious negative relation to parents' excessive interference, protection, punishment, strictness, partiality and mother's refusal [20]. Wang and Ding's result [21] further shows that the more comprehension and care from parents, the more positive emotions children will experience, with better life satisfaction and higher general well-being. On the contrary, if parents do not interfere or interfere too much, children will experience less well-being. Whereas, parents who with higher education background will give more warmth and comprehension to children. Otherwise, parents usually choose autocracy or doting [22]. Perhaps it is because parents with higher education background or engaging in scientific technologies or culture can treat things and problems more objectively and reasonably, can more respect others and pay more attention to the feel inside of others, can educate children with scientific attitude and suitable method. At the same time, they ask more for children's school work, too. So in our research friendship satisfaction and positive emotions of adolescents whose parents are engaging in scientific technologies or culture or have higher education background are higher than those of adolescents whose parents are Farmers or with lower education ba- 
ckground. For the same reason, school satisfaction of adolescents is contrary. On the other hand, parents are labors or intellects, with great work pressure and tension. So they take less care of children compared with Farmers. Accordingly, family satisfaction of adolescents whose parents are labors or intellects is lower than that of adolescent whose parents are Farmers. Overall, for the healthy growth of children, it will be meaningful for parents to strengthen their self-cultivation and improve their self-quality, to know more of physiologic and mental characteristics of children, to learn more of scientific methods to educate children.

\section{REFERENCES}

[1] Wu, M.X. (2000) Theoretical development of subjective well-being in the west for 30 years. Journal of Developments in Psychology (China), 4, 23-28.

[2] Diener, E. (1984) Subjective well-being. Journal of Psychological Bulletin, 95(3), 542-575.

[3] Ren, Z.H. and Ye, Y.-D. (2006) Review on domestic and overseas researches of affecting factors of subjective well-being. Journal of Fujian Normal University (Philosophy and Social Sciences Edition) (China), 139(4), 152-158.

[4] Mandler, J.M. (1992) How to build a baby: II. Conceptual primitives. Journal of Psychological Review, 99(4), 587-604.

[5] Zhang, X.G, He, L.G. and Zheng, X. (2004) Adolescent students life satisfaction: Its construct and scale development. Journal of Psychological Science (China), 27(5), 1257-1260.

[6] Gu, Y.Y. and Luo, Y.J. (2009) Neural mechanism of subjective well-being. Journal of Advances in Psychological Science (China), 17(5), 957-963.

[7] Wang, X.D., Wang, X.L. and Ma, H. (1999) Handbook of mental health scale. Enlarged Edition, Chinese Mental Health Magazine, Beijing.

[8] Huebner, E.S., Drane, W. and Valois, R.F. (2002) Levels and demographic correlates of adolescent life Satisfaction Reports. Journal of School Psychology International, 21(3), 281-292.

[9] Xiao, J.W. and Shi, G.X. (2005) A relative study of the senior high school students daily event, mental health, and sense of happiness. Journal of Hebei Normal University Educational Science Edition, 7(2), 75-78.
[10] Zhao, S.Y. (2006) A survey on subjective well-being of students in key high schools. Chinese Journal of Special Education, 69(3), 59-62.

[11] Dew, T. and Huebner, E.S. (1994) Adolescents' perceived quality of life: An exploratory investigation. Journal of School Psychology, 32(2), 185-199.

[12] Huebner, E.S., Drane, W. and Valois, R.F. (2000) Levels and demographic correlates of adolescent life satisfaction repots. Journal of School Psychology International, 21(3), 281-292.

[13] Gilman, R. and Huebner, E.S. (2003) A review of life satisfaction research with children and adolescents. Journal of School Psychology Quarterly, 2, 107-116.

[14] Zang, X.-G., He, L.-G. and Jiao, L. (2007) The structural relationship among big five personality, demography variable and SWB. Journal of Psychological Development and Education (China), (1), 46-53.

[15] Su, X. (2008) Relationship between subjective wellbeing and academic achievement of junior middle school students. Journal of Henan Medical College for Staff and Workers, 20(3), 278-276.

[16] Zhang, X.Q. and Sun, C.X. (2009) A study subjective well-being of secondary school students. Journal of Youth \& Juvenile Research (China), 69(1), 20-25.

[17] Wang, G., Zang, D.J. and Liang, L. (2008) The development characteristics of high school students' subjective well-being and its relationship with academic self. Journal of Chinese Special Education, 101(11), 90-95.

[18] Ma, Y. and Liu, D.Z. (2005) Middle school students learning subjective well-being and its major influencing factors. Journal of Psychological Development and Education (China), (1), 74-79.

[19] Lou, W.Q and Chi, I. (2002) The Stressors and psychological well-being of senior secondary school students. Journal of Psychological Science (China), 23(2), 156-159.

[20] Hu, J., Ji, T.S. and Feng, F.L. (2002) A study of relationship between parental rearing patterns and general well-being of college students. Journal of Chinese Health Psychology, 10(1), 16-17.

[21] Wang, J.S and Ding, X.H. (2003) Relationship between subjective well-being and coping style of junior school student. Journal of Chinese Public Health, 11(2), 11811182.

[22] Song, H.M., Shen, J.H., and Li, B.W. and Xiang, X.Z. (2004) A study of relationship between parental education and parental rearing patterns of college students. Journal of Medical Journal of Chinese People Health, 16(9), 571-572. 